\title{
Basal chromospheric flux and Maunder Minimum-type stars: the quiet-Sun chromosphere as a universal phenomenon (Research Note)
}

\author{
K.-P. Schröder ${ }^{1}$, M. Mittag ${ }^{2}$, M. I. Pérez Martínez ${ }^{1}$, M. Cuntz ${ }^{3}$, and J. H. M. M. Schmitt ${ }^{2}$ \\ 1 Departamento de Astronomía, Universidad de Guanajuato, Apartado Postal 144, 36000 Guanajuato, Mexico \\ e-mail: kps@astro.ugto.mx \\ 2 Hamburger Sternwarte, Universität Hamburg, Gojenbergsweg 112, 21029 Hamburg, Germany \\ ${ }^{3}$ Department of Physics, Science Hall, University of Texas at Arlington, Arlington, TX 76019-0059, USA
}

Received 29 October 2011 / Accepted 12 February 2012

\begin{abstract}
Aims. We demonstrate the universal character of the quiet-Sun chromosphere among inactive stars (solar-type and giants). By assessing the main physical processes, we shed new light on some common observational phenomena.

Methods. We discuss measurements of the solar Mt. Wilson $S$-index, obtained by the Hamburg Robotic Telescope around the extreme minimum year 2009, and compare the established chromospheric basal Ca II K line flux to the Mt. Wilson $S$-index data of inactive ("flat activity") stars, including giants.

Results. During the unusually deep and extended activity minimum of 2009, the Sun reached $S$-index values considerably lower than in any of its previously observed minima. In several brief periods, the Sun coincided exactly with the $S$-indices of inactive ("flat", presumed Maunder Minimum-type) solar analogues of the Mt. Wilson sample; at the same time, the solar visible surface was also free of any plages or remaining weak activity regions. The corresponding minimum Ca II K flux of the quiet Sun and of the presumed Maunder Minimum-type stars in the Mt. Wilson sample are found to be identical to the corresponding Ca II K chromospheric basal flux limit.

Conclusions. We conclude that the quiet-Sun chromosphere is a universal phenomenon among inactive stars. Its mixed-polarity magnetic field, generated by a local, "fast" turbulent dynamo finally provides a natural explanation for the minimal soft X-ray emission observed for inactive stars. Given such a local dynamo also works for giant chromospheres, albeit on longer length scales, i.e., $l \propto R / g$, with $R$ and $g$ as stellar radius and surface gravity, respectively, the existence of giant spicular phenomena and the guidance of mechanical energy toward the acceleration zone of cool stellar winds along flux-tubes have now become traceable.
\end{abstract}

Key words. Sun: chromosphere - Sun: faculae, plages - Sun: dynamo - Sun: corona - stars: chromospheres - stars: activity

\section{Introduction}

The chromospheric and coronal heating problem is one of the unsolved questions in the realm of solar and stellar physics. The underlying dominant physical processes have not been identified in detail, and we are far from having fully understood the quite diverse observational evidence pertaining to the observed plethora of chromospheric and coronal emissions of cool stars. The recent lull of solar activity has raised the question to what extent we can regard the quiet-Sun and its chromosphere as representative for other inactive or low-activity stars, particularly the so-called Maunder Minimum ("flat activity") Mt. Wilson stars (Baliunas et al. 1995), or the many inactive giants with emission line fluxes close to the basal chromospheric flux limit as discussed by, e.g., Pérez Martínez et al. (2011). Can we even obtain insight into the guiding mechanisms of extended chromospheres and cool winds of giants by analyzing the quiet Sun?

An important milestone in our understanding of stellar chromospheres was set by Schrijver (1987) and Schrijver et al. (1989, 1992), who identified a so-called basal component in the chromospheric line emission, notably in the $\mathrm{Ca}$ II $\mathrm{H}+\mathrm{K}$ and $\mathrm{Mg}$ II $h+k$ lines, which is relatively small compared to and independent of the large observed variations of the chromospheric emission commonly attributed to stellar magnetic activity. This concept of a basal flux was subsequently confirmed by several authors, including Strassmeier et al. (1994) and Pasquini et al. (2000). A plausible physical explanation was also readily offered through the dissipation of non-magnetic energy, proposed to exist in the form of acoustic waves. Hydrodynamic models of these waves, including their propagation, shock formation and dissipation, indeed showed the same dependence of the emitted flux on the stellar effective temperature $T_{\text {eff }}$ as observed for the basal chromospheric flux, which is $T_{\text {eff }}^{8}$ (e.g., Buchholz et al. 1998). Additionally, in a quantitative study of a distinct Maunder Minimum-type star in the Mt. Wilson sample, i.e., $\tau$ Ceti, Rammacher \& Cuntz (2003) were able to reproduce the basal chromospheric flux in terms of acoustic wave energy dissipation with high accuracy.

Nevertheless, this simple picture was seriously challenged when also a minimum flux in the soft X-ray emission was detected, even for stars suspected to be Maunder Minimum ("flat activity") candidates of the Mt. Wilson sample (Schmitt 1997; Saar 1998; Judge et al. 2004). According to detailed theoretical reasoning (e.g., Stępień \& Ulmschneider 1989; Hammer \& Ulmschneider 1991), acoustic waves cannot account for the observed X-ray emission, and therefore the exact physical 


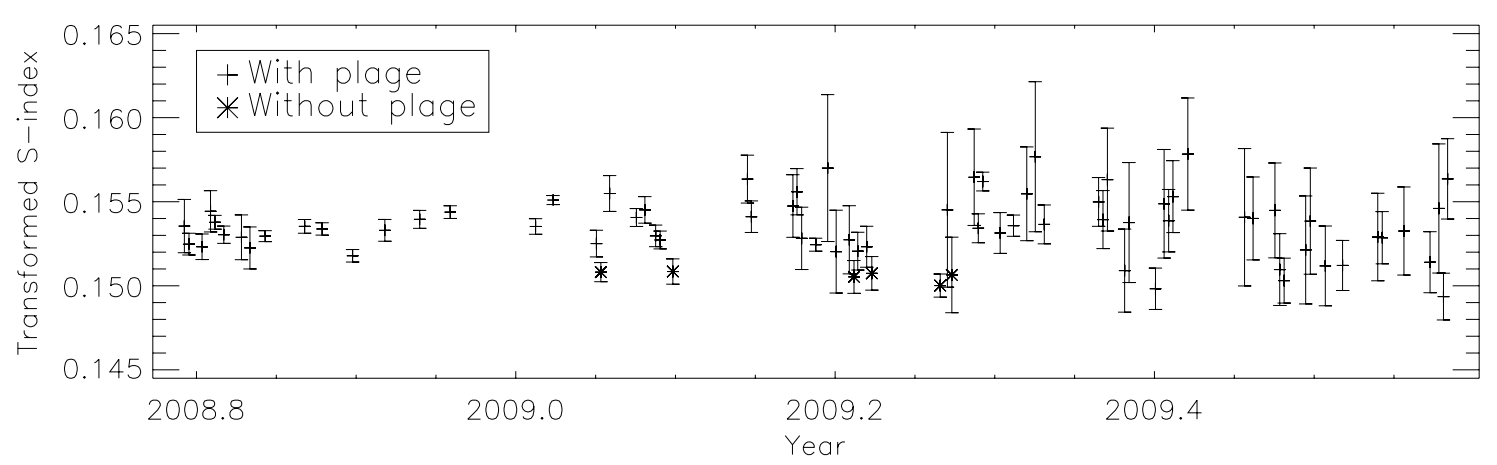

Fig. 1. Mt. Wilson $S$-index measurements obtained for the Sun by the Hamburg Robotic Telescope and HEROS Échelle spectrograph during the extraordinary activity minimum 2008/2009. A minimum $S$ value of 0.150 is reached on several plage-free days. Error bars are mainly due to noise, which varies with temperature and humidity.

process(es) leading to the basal chromospheric flux still remain a topic of intense debate (e.g., Judge \& Cuntz 1993; Judge \& Carpenter 1998).

For evolved stars the prevalence of acoustic rather than magnetic heating appears to be consistent with basic ideas on stellar angular momentum evolution: when solar-type stars evolve away from the main-sequence, their rotation slows down beyond what should be expected from the increase in the moment of inertia caused by changes in the internal mass distribution (i.e., Gray 1991; Schrijver 1993; Schrijver \& Pols 1993; Keppens et al. 1995; Charbonneau et al. 1997). Hence, the stellar angular momentum subsides due to magnetic braking resulting from the onset of massive stellar winds and therefore little magnetic activity is expected to persist in evolved stars.

Based on long-term time series observations of chromospheric Ca II K emission, i.e., the Mt. Wilson project (Baliunas et al. 1995), stars identified to exhibit very low $S$-index levels, i.e., without regular, cyclic changes, thus considered as "flat", appear to be in a state comparable to the solar Maunder Minimum. During the period of more than 30 years covered by the Mt. Wilson project, which also included several solar activity minima, the Sun kept a distinct positive distance from these low $S$-levels; yet is by wide standards not considered a highly active star. Therefore, it remained unclear, how inactive a Maunder Minimum Sun really is. From historic observations we are aware of a prevailing, though not always complete, absence of sunspots, but this may not be representative for Ca II plages.

A rough comparison shows that at activity minimum the solar chromosphere is relatively close to its basal flux. This is, however, difficult to verify for other Maunder Minimum stars, since any quantitative interpretation of the Mt. Wilson $S$-indices depends on the exact knowledge of the flux in two UV continuum windows used to normalize the chromospheric line emission fluxes. Hence, there may be long-suspected similarities between Maunder Minimum Mt. Wilson stars, the basal chromospheric flux and the quiet solar chromosphere (see Judge \& Saar 2007, for measurements in the UV and X-ray regimes), but the final proof has still been missing. In addition, the rich presence of mixed-polarity magnetic field in the quiet Sun has always called into question whether its characteristic physics would be a good proxy for truly inactive stars, especially giant stars.

In this paper, we will present the final clue regarding the universality of the quiet-Sun chromosphere as the underlying phenomenon to both Maunder Minimum-type stars and basal chromospheric flux stars. We then will give a quantitative interpretation of the Mt. Wilson $S$-index data in terms of projecting the basal chromospheric $\mathrm{Ca}$ II $\mathrm{K}$ flux into the
$S-(B-V)$ diagram. Finally, we will discuss the aforementioned apparent contradictions in the light of current knowledge about the physical processes in the quiet-Sun chromosphere.

\section{The solar S-index at Maunder Minimum level}

For both historic and instrumental reasons, the Mt. Wilson $S$-index is defined as the flux ratio between the central $1 \AA$ wide, mostly chromospheric $\mathrm{Ca}$ II $\mathrm{H}$ and $\mathrm{K}$ line emissions, $F_{\mathrm{H}}$ and $F_{\mathrm{K}}$, and two nearby reference bands of $20 \AA$ of UV quasicontinuum flux, $F_{\mathrm{R}}$ and $F_{\mathrm{V}}$ at 3901 and $4001 \AA$, i.e., $S=\alpha\left(F_{\mathrm{H}}+\right.$ $\left.F_{\mathrm{K}}\right) /\left(F_{\mathrm{R}}+F_{\mathrm{V}}\right)$; see Baliunas et al. (1995). According to Duncan et al. (1991), the nightly calibration factor $\alpha$ is about constant, i.e., $\alpha=2.4$. By contrast, $S$-indices based on present, normalized spectra need to be scaled by a factor of approximately 19 (see Hall et al. 2007, who derived a value 19.2), to compare the new $S$-indices with the original Mt. Wilson $S$-indices, $S_{\text {Mwo. In }}$ a first test phase of the Hamburg Robotic Telescope (HRT) and its Échelle spectrograph HEROS (Heidelberg Extended Range Optical Spectrograph; see Hempelmann et al. 2005, for a detailed description of the HRT including its hardware) in the time frame between October 2008 and July 2009 we obtained normalized spectra of a sample of stars and the Sun spanning the whole activity scale with a spectral resolution of $R \approx 20000$. Using specifically observations of 29 selected, well-observed and stable Mt. Wilson stars with $S_{\text {MwO }}$ between 0.15 and 0.7 , we determined the following, very tight linear relation between

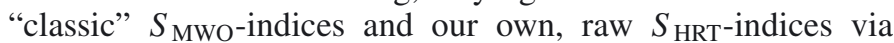
$S_{\text {MWO }}=(0.0294 \pm 0.0040)+(19.79 \pm 0.56) \cdot S_{\text {HRT }}$, which allows us to compare our $S$-indices to the large number of MWO $S$-indices (Duncan et al. 1991).

During October 2008 and July 2009, we observed the Sun on 73 occasions, usually by taking day-sky spectra and twice by taking lunar spectra. Fortuitously, this observing series coincides with the exceptionally long and deep past solar minimum. All HEROS spectra were reduced with the fully automatic reduction pipeline of the HRT (see Mittag et al. 2010). The day-sky spectra were furthermore corrected for Mie and Rayleigh scattering, with a lunar spectrum serving as reference. To estimate the error of this correction, the continuum flux of the day-sky spectra, after correction, and the reference moon spectrum were compared. The remaining differences were very small, indeed, in the range of $0.2-1.8 \%$, on average approximately $0.6 \%$.

Our individual solar measurements are shown as a time series in Fig. 1; the measured $S$-index values are distributed between 0.150 and 0.157 , with an average solar $S$-index of $S_{\text {MWO }}=0.153 \pm 0.005$ in that period. By contrast, the solar 


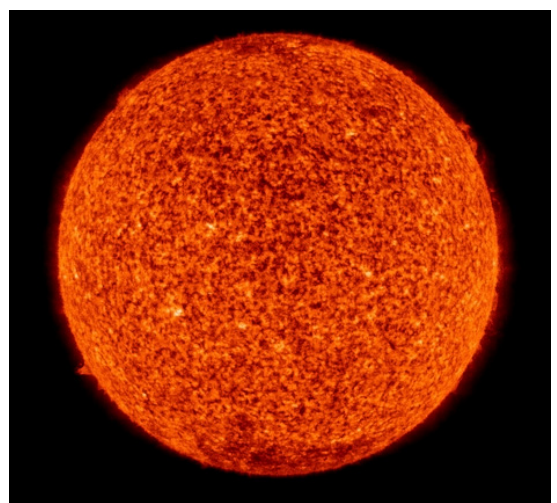

Fig. 2. Plage-free solar chromosphere on February 6, 2009, taken by $\mathrm{SOHO}$ at $304 \mathrm{~nm}$.

$S$-index measurements obtained in the context of the Mt. Wilson project showed that the solar $S$-index varied between about 0.16 (normal activity minima) and 0.22 (maxima) between 1966 and 1993 (Baliunas et al. 1995). The abnormally low value of the solar $S_{\text {MWO }}$ found by us in 2009 must be real and caused by the unusually deep solar minimum, rather than by errors in the above-stated transformation from $S_{\text {HRT }}$ to $S_{\text {MWO }}$, since the observed, average offset $(>0.007)$ is a lot larger than the respective uncertainty of the transformation (0.004).

In summary, we argue that our solar $S$-index measurements from October 2008 to July 2009 are indeed lower than any of the solar $S$-indices measured in the context of the Mt. Wilson project, ever. Most interestingly, these values are very close to the $S$-indices of some solar-type, "flat activity" Mt. Wilson sample stars, i.e., HD $43587(B-V=0.61)$ and HD 143761 $(B-V=0.60$; see Baliunas et al. 1995). The absence of any higher activity for over 30 years make these stars excellent Maunder Minimum candidate stars, and furthermore, because their $T_{\text {eff }}$ values are very similar to those of the Sun, their $S$-indices can be directly compared to the solar values.

Since sunspots were very rare during our specific observing period, there is little correlation between the $S$-index values and the sunspot numbers. In particular, there are many $S$-index measurements on days where the sunspot number was zero. However, for the solar Ca II emission the sunspots are of lesser importance, whereas the presence of any plages (faculae) would directly impact the measured $S$-index. Indeed, the lowest obtained solar $S$-values of nearly 0.150 occurred only on days without any plages at all (see Fig. 2), as already proposed by White et al. (1992).

\section{S-index and basal chromospheric flux}

Next we explore the relationship between the $S$-index and the basal chromospheric flux for main-sequence stars and giants. Figure 3, which displays the distribution of $S_{\text {MWO }}$ (Duncan et al. 1991) for main-sequence stars (black dots) and giant stars (shaded dots) over $B-V$, strongly suggests that the lower boundaries of the respective distributions correspond to inactive stars with pure basal chromospheric emission. Main-sequence stars of spectral types $\mathrm{K}$ and $\mathrm{M}$ toward the red end are increasingly more active. By contrast, only G-type giants show a significant amount of chromospheric activity (e.g., Pérez Martínez et al. 2011). It is also at the basal flux edge (around $S=0.15$ ) of G-type main-sequence stars, where the Maunder Minimum-type "flat activity" Mt. Wilson stars as well as the plage-free Sun are found. However, because quasi-continua and Ca II photospheric line-cores are both included in the definition of $S$ and also

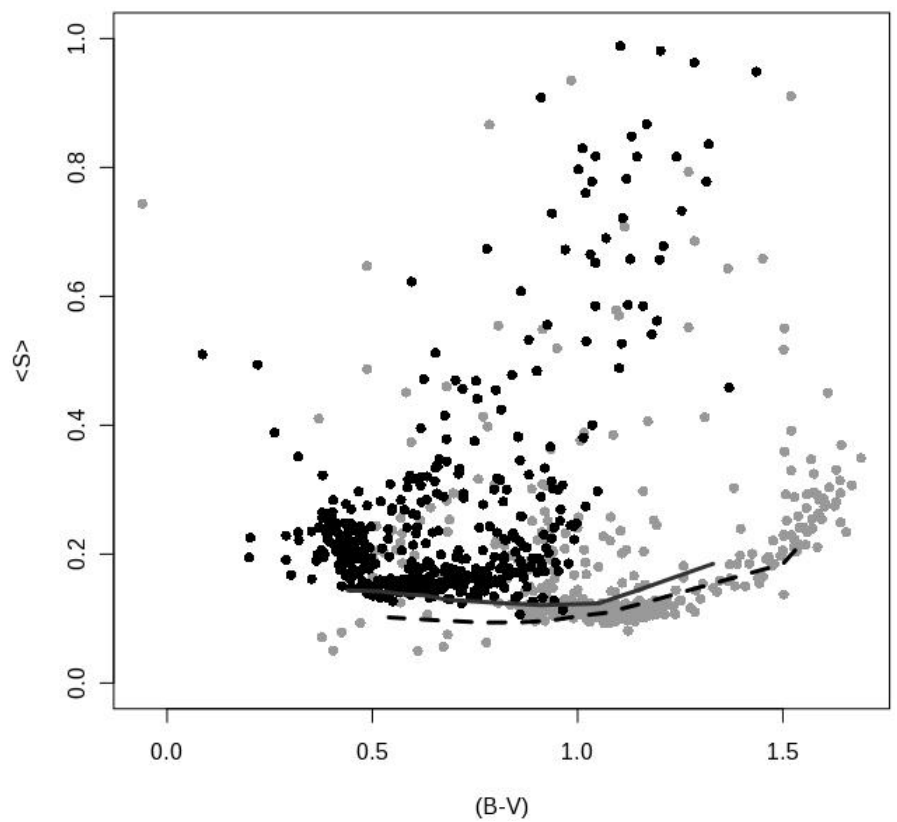

Fig. 3. Observed distribution of stellar Mt. Wilson $S$-indices over $B-V$, together with the location of basal chromospheric Ca II flux (see text). Although exhibiting the same basal flux, giants (shaded dots) show a systematic offset in $S$ against main-sequence stars (black dots) because their photospheric spectral properties are gravity-sensitive (dashed and solid line, respectively). Most notably, Mt. Wilson Maunder Minimumtype stars and the plage-free solar $S$-values coincide with the Ca II basal flux limit.

depend on the stellar gravity, giants at basal flux levels are offset from the respective main-sequence to slightly lower $S$-values.

With a detailed revision of the Ca II K basal chromospheric surface line flux, based on new spectroscopic observations and non-LTE photospheric models, which are still being developed, we employ a simple relationship based on Mg II $h+k$ line emission, which is much easier to measure and quantify. Using IUE spectra of a set of inactive cool giant stars, we previously derived the following relation between the "basal" chromospheric surface flux in the $\mathrm{Mg}$ II $h+k$ lines and effective temperature $T_{\text {eff }}: \log F_{\text {Mg II }}=7.33 \log T_{\text {eff }}-21.75$ (in cgs units; see Pérez Martínez et al. 2011, and references therein, for details). Earlier work on main-sequence stars arrived at a very similar result, which again shows that the basal chromospheric flux itself (within its uncertainties) does not depend on gravity. Furthermore, according to Schrijver et al. (1992), Mg II $h+k$ and $\mathrm{Ca}$ II $\mathrm{H}+\mathrm{K}$ chromospheric line fluxes differ by a fixed factor, which is $0.78 \pm 0.05$. By dividing the $\mathrm{Mg}$ II fluxes from the above given relationship by this factor, we are able to derive $\mathrm{Ca}$ II $\mathrm{H}+\mathrm{K}$ basal surface fluxes, allowing us to compute "basal" $S$-indices.

Compared to the work by Strassmeier et al. (1994), who determined $\log F_{\text {Ca II }}=8.0 \log T_{\text {eff }}-24.8$ directly from Ca II line emissions of cool giant stars, our basal flux values turn out to be higher by a factor of 2 to 4 ; they are of the same order as the photospheric line core fluxes as confirmed by Fig. 4. This plot compares the Ca II K line core of HD 109379, a basal flux giant, and its chromospheric emission at basal flux level to a synthetic spectrum given by a stellar atmosphere model obtained by the Phoenix code (see Hauschildt \& Baron 2005; with $T_{\text {eff }}=5800 \mathrm{~K}$ and $\log g=2.5$ ) to make a photospheric template and an accurate, physical surface flux scale. By contrast, the scale used by Strassmeier et al. (1994) relied on historic work from the 1970s. The same appears to apply to the work by White et al. (1992), 


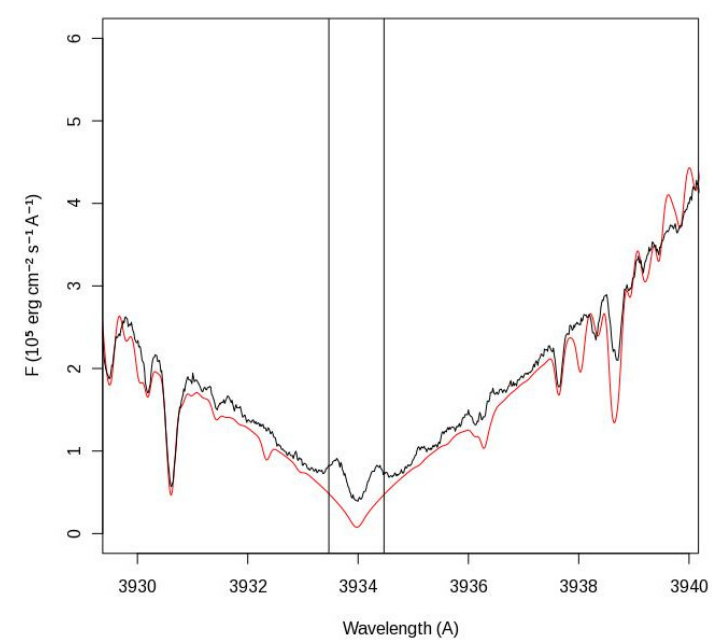

Fig. 4. Ca II K line core of HD 109379, a giant star, showing its basal chromospheric line emission in nearly equal proportion to the underlying photospheric line flux, represented by a Phoenix model atmosphere spectrum (Hauschildt \& Baron 2005).

who found that the Ca II K line flux of inactive solar-type chromospheres would fall much below that of the average quiet Sun.

To properly translate the basal chromospheric Ca II K flux into $S_{\text {MWO }}$, the underlying photospheric fluxes in the Ca II line centers, $F_{\mathrm{H}}$ and $F_{\mathrm{K}}$, need to be known, as well as the abovementioned UV quasi-continuum fluxes $F_{\mathrm{R}}$ and $F_{\mathrm{V}}$. For a variety of effective temperatures and two representative sets of gravity (i.e., $\log g=4$ for the old, least active main-sequence stars and $\log g=2$ for LC III giants) we derived these quantities from Phoenix model spectra. The resulting raw values of "basal $S$ " were multiplied by 19 to match the $S_{\mathrm{MWO}}$-scale. Finally, effective temperatures were translated into $B-V$ colors using the tables of Schmidt-Kaler (1982). The resulting "basal" $S$-values are depicted in Fig. 3 (solid and dashed lines, respectively). Since both quasi-continua and Ca II photospheric line cores, are gravity-sensitive, giants at the basal flux limit are offset from the main-sequence stars at the basal flux limit to slightly lower $S$-values. It is near the main-sequence basal flux $S$-values (around 0.15) that we find the Maunder Minimum type "flat activity" stars as well as the plage-free Sun.

\section{A local "fast" dynamo: new answers to old questions?}

Through the kind cooperation of our central star in 2009, we found direct proof that it is the quiet-Sun chromosphere which is behind the formation of basal chromospheric flux, and that a Maunder Minimum-type star exhibits a truly quiet-Sun chromosphere. Therefore, we may now conclude that the quiet Sun is not just similar to inactive stars, but it even represents the universal phenomenon that produces the basal flux of all inactive cool stars with some adjustments for low gravity pertaining to giant stars (see discussion). With this insight in mind, can we now learn something new by looking at the physical processes typical for the quit-Sun chromosphere?

The rich presence of mixed-polarity flux in the quiet Sun nurtures phenomena like spicules and bright points, which are related to rising flux-tubes and their dynamic interaction with convection, and a minimal coronal emission of soft X-rays from solar coronal holes and even Maunder Minimum stars (Schmitt 1997). It was long thought that this magnetic presence was a side product of the main stellar activity through magnetic dissipation of the strong, rising local fields. But the level of magnetic fluxtube density in the quiet-Sun surface does not change with the overall activity of the Sun, while we would expect it to show some following-tendency with the solar activity on a time-scale of months or years. Hence, its nature must be entirely independent of what we understand as magnetic activity (local, strong fields, rising up from the bottom of the convection zone where a global dynamo produced them).

Recently, Vögler \& Schüssler (2007) have presented a magnetohydrodynamic (MHD) simulation for the quiet-Sun surface, which yields a mixed-polarity field, generated by a local "fast" turbulent dynamo operating only in the thin uppermost layer of the convection zone. The principle of such a non-cyclic mechanism to create a light distribution of random magnetic field has already been suggested by Saar (1998), who also anticipated its potential in explaining the soft X-ray flux and transition region emission concerning inactive chromospheres, where pure acoustic wave heating would well account for only the basal line flux. The simulation of Vögler \& Schüssler (2007) starts with a weak seed field, and soon a fairly well-defined saturation level of the magnetic energy density of a few percent of the total kinetic energy is attained.

This result is nicely consistent with the relative flux contribution of the solar bright points to the total light in both MHD simulations (Unruh et al. 2009) and high-resolution observations (by the SUNRISE balloon-borne observatory, Riethmüller et al. 2010). In Ca II K, bright points appear to contribute only about $10 \%$ of the quiet-Sun chromospheric flux. The by far largest contribution to the quiet-Sun chromospheric line emission seems to arise from a highly dynamic process (Wöger et al. 2006), which operates on a time-scale significantly shorter than the underlying convection, and which we may therefore attribute to the action of acoustic waves; see, e.g., Cuntz et al. (2007) for recent solar acoustic heating models.

Hence, both present quiet-Sun observations and detailed hydrodynamic case studies of non-active chromospheres with acoustic wave dissipation strongly hint at kinetic energy to be responsible for creating the major part of the basal chromospheric flux. However, this concept alone cannot explain the coronal soft $\mathrm{X}$-ray emission from above non-active areas. However, invoking the idea of an embedded fairly constant share of magnetic field, this puzzle is finally solved: the typical minimum soft X-ray surface flux, as found in coronal holes and for Maunder Minimumtype stars like $\tau$ Cet, is close to $10^{4} \mathrm{erg} \mathrm{cm}^{-2} \mathrm{~s}^{-1}$ (Schmitt 1997; Judge et al. 2004). That is nearly two orders of magnitude below the total basal chromospheric cooling flux of all emission lines combined, consistent with an energy content of the magnetic field of a few percent of the kinetic energy of the turbulent chromosphere. According to Pevtsov et al. (2003), the minimum X-ray flux requires a magnetic field of about only 10 Gauss, which easily falls within the yield of the MHD simulation of Vögler \& Schüssler (2007).

We therefore conclude that local dynamo action finally resolves the long-standing puzzle of the origin of a minimum X-ray flux, in the absence of any ("classical") stellar activity as we used to know it, i.e., in the absence of active regions.

\section{Discussion and conclusions}

In view of the universality of physics, it is a sensible speculation that the same local "fast" dynamo would produce a similar content of mixed-polarity magnetic flux-tubes in giant chromospheres. Since the local "fast" dynamo is entirely different 
from the classic $\alpha-\Omega$ dynamo and therefore independent of any global dynamo action, it should provide a universal presence of flux-tubes at a fairly well-defined saturation level, for every cool giant, and not just for their highly active tail-end (e.g., Konstantinova-Antova et al. 2010). Indeed, according to Brandenburg (2011), these locally generated magnetic fields do not vary much with Prandtl number.

There is one special aspect for giant stars, however, which requires more careful consideration: here we must expect all scale lengths $l$ to be a lot longer because of the much lower gravity, following Schwarzschild's simple relation $l \propto R / g$ with $R$ and $g$ as stellar radius and surface gravity, respectively (Schwarzschild 1975). In this concept of a growing extent of chromospheres and their embedded features with lower values of $g$, we would also expect the occurrence of giant spicules. Indeed, these have already been suspected two decades ago based on data gathered during spectroscopic eclipses of HR 2554 and HR 6902 (Schröder \& Hünsch 1992).

Furthermore, if the local "fast" turbulent dynamo were still able to operate in an environment of extremely low gravity, particularly concerning the photospheres of K-type supergiants like $32 \mathrm{Cyg}$, the resulting presence of local, random magnetic fields would also provide us a plausible explanation for transient, prominence-like clouds of chromospheric material, long known from spectroscopic eclipse observations of $\zeta$ Aurigae systems by "satellite lines" in Ca II K (e.g., Wilson 1960). Schröder (1983) found a large cloud in an IUE spectra series of the 1981 eclipse of 32 Cyg, 1/6 giant radius off-limb, by its Rayleigh scattering and concluded that a magnetic field of about 4 Gauss (if $T_{\text {eff }}=3000 \mathrm{~K}$ ) would suffice to balance its thermal energy. With an updated choice of a chromospheric temperature of $6000 \mathrm{~K}$, this would amount to about 6 Gauss, which still lies very reasonably within the the means of the quoted local dynamo.

A supply of flux-tubes to giant chromospheres would also provide a natural explanation for the vertical transport of mechanic flux from the kinetic energy reservoir of the turbulent chromosphere up into the cool wind acceleration region, as suggested by Schröder \& Cuntz (2005) and Suzuki (2007). The problem is that acoustic waves are expected to be insufficient because they dissipate their energy relatively close to the star (e.g., Cuntz 1990), but vertical magnetic structure may constitute a venue for this kind of energy transport. Hence, we conclude that a number of hitherto unresolved problems can be seen in an entirely new light, and apparent contradictions disappear if the latest insight into the principal and apparently quite universal physics of the quiet-Sun chromosphere is considered.

Another very interesting and direct result of our 2009 solar $S$-index measurements is the correspondence of the established Maunder Minimum $S$-level of 0.150 with the complete absence of even plages, hence of any sign of activity regions. This implies that during a Maunder Minimum, surface activity completely vanishes for most of the time or must be very subtle. The question remains as to why and for how long.

Acknowledgements. This work was supported by travel funds from the bilateral Conacyt-DFG grant No. 147902. Furthermore, we are most grateful for the funding of the robotic telescope project by the University of Hamburg and the Deutsche Forschungsgemeinschaft (instrumentation), by the Universities of Guanajuato and Liège, and by the State of Guanajuato (infrastructure at the new location in Guanajuato, Mexico). Finally, we wish to thank our colleague Peter Hauschildt for providing us with a large set of Phoenix models.

\section{References}

Baliunas, S. L., Donahue, R. A., Soon, W. H., et al. 1995, ApJ, 438, 269

Brandenburg, A. 2011, ApJ, 741, 92

Buchholz, B., Ulmschneider, P., \& Cuntz, M. 1998, ApJ, 494, 700

Charbonneau, P., Schrijver, C. J., \& MacGregor, K. B. 1997, in Cosmic Winds and the Heliosphere, ed. J. R. Jokipii, C. P. Sonett, \& M. S. Giampapa (Tucson: University of Arizona Press), 677

Cuntz, M. 1990, ApJ, 353, 255

Cuntz, M., Rammacher, W., \& Musielak, Z. E. 2007, ApJ, 657, L57

Duncan, D. K., Vaughan, A. H., Wilson, O. C., et al. 1991, ApJS, 76, 383

Gray, D. F. 1991, in Angular Momentum Evolution of Young Stars, ed. S. Catalano, \& J. R. Stauffer (Dordrecht: Kluwer), 183

Hall, J. C., Henry, G. W., \& Lockwood, G. W. 2007, AJ, 133, 2206

Hammer, R., \& Ulmschneider, P. 1991, in Mechanisms of Chromospheric and Coronal Heating, ed. P. Ulmschneider, E. R. Priest, \& R. Rosner (Berlin: Springer), 344

Hauschildt, P., \& Baron, E. 2005, Mem. S. A. It. Suppl., 7, 140

Hempelmann, A., González-Pérez, J. N., Schmitt, J. H. M. M., \& Hagen, H. J. 2005, in Cool Stars, Stellar Systems, and the Sun 13, ed. F. Favata, G. A. J. Hussain, \& B. Battrick, ESA SP, 560, Vol. II, 643

Judge, P. G., \& Carpenter, K. G. 1998, ApJ, 494, 828

Judge, P. G., \& Cuntz, M. 1993, ApJ, 409, 776

Judge, P. G., \& Saar, S. H. 2007, ApJ, 663, 643

Judge, P. G., Saar, S. H., Carlsson, M., \& Ayres, T. R. 2004, ApJ, 609, 392

Keppens, R., MacGregor, K. B., \& Charbonneau, P. 1995, A\&A, 294, 469

Konstantinova-Antova, R., Aurière, M., Charbonnel, C., et al. 2010, A\&A, 524, A57

Mittag, M., Hempelmann, A., González-Pérez, J. N., \& Schmitt, J. H. M. M. 2010, Adv. Astron., 2010, 101502

Pasquini, L., de Medeiros, J. R., \& Girardi, L. 2000, A\&A, 361, 1011

Pérez Martínez, M. I., Schröder, K.-P., \& Cuntz, M. 2011, MNRAS, 414, 418

Pevtsov, A. A., Fisher, G. H., Acton, L. W., et al. 2003, ApJ, 598, 1387

Rammacher, W., \& Cuntz, M. 2003, ApJ, 594, L51

Riethmüller, T. L., Solanki, S. K., Martínez Pillet, V., et al. 2010, ApJ, 723, L169

Saar S. H. 1998, in Cool Stars, Stellar Systems and the Sun 10, ed. R. A. Donahue, \& J. A. Bookbinder (San Francisco: ASP), ASP Conf. Proc., 154, 211

Schmidt-Kaler, Th. 1982, in Landolt-Börnstein, ed. K. Schaifers, \& H. H. Voigt, New Series, Group 6, Vol. 2b (Berlin: Springer)

Schmitt, J. H. M. M. 1997, A\&A, 318, 215

Schrijver, C. J. 1987, A\&A, 172, 111

Schrijver, C. J. 1993, in Inside the Stars, ed. W. W. Weiss, \& A. Baglin (San Francisco: ASP), IAU Coll., 137, ASP Conf. Proc., 40, 591

Schrijver, C. J., \& Pols, O. R. 1993, A\&A, 278, 51

Schrijver, C. J., Dobson, A. K., \& Radick, R. R. 1989, ApJ, 341, 1035

Schrijver, C. J., Dobson, A. K., \& Radick, R. R. 1992, A\&A, 258, 432

Schröder, K.-P. 1983, A\&A, 124, L16

Schröder, K.-P., \& Cuntz, M. 2005, ApJ, 630, L73

Schröder, K.-P., \& Hünsch, M. 1992, A\&A, 257, 219

Schwarzschild, M. 1975, ApJ, 195, 137

Stępień, K., \& Ulmschneider, P. 1989, A\&A, 216, 139

Strassmeier, K. G., Handler, G., Paunzen, E., \& Rauth, M. 1994, A\&A, 281, 855 Suzuki, T. K. 2007, ApJ, 659, 1592

Unruh, Y. C., Solanki, S. K., Schüssler, M., Vögler, A., \& Garcia-Alvarez, D. 2009, in Cool Stars, Stellar Systems and the Sun 15, ed. E. Stempels, AIP Conf. Ser., 1094, 768

Vögler, A., \& Schüssler, M. 2007, A\&A, 465, L43

White, O. R., Skumanich, A., Lean, J., Livingston, W. C., \& Keil, S. L. 1992, PASP, 104, 1139

Wilson, O. C. 1960, in Stellar Atmospheres, ed. J. L. Greenstein, 436

Wöger, F., Wedemeyer-Böhm, S., Schmidt, W., \& von der Lühe, O. 2006, A\&A, 459, L9 